\title{
SMART GARBAGE ALERT SYSTEM USING MACHINE LEARNING
}

\author{
V. Srinithi Santhana Lakshmi, P. Sujatha, M. Swathi \\ Ms. P. Premadevi, Associate professor \\ Department of CSE \\ Dhanalakshmi Srinivasan College of Engineering And Technology, Chennai
}

\begin{abstract}
The main aim project is to develop a smart intelligent garbage alert system for a proper garbage management. Our project proposes a smart AI dustbin which is Collect the Real time dataset of what's in the Garbage and Classify the Waste. Prepare the Dataset for an Image classification. Train the Neural Network and Create a Tensor Flow Model and Deploy the Model. After it is also interfaced with hardware using ultrasonic sensor. The sensor values are read and the values are displayed in the LCD. Hence, garbage management is done by using both image processing and embedded system. Based on the image processing output, the respective degradable and non-degradable dustbins are opened by showing relay operations.
\end{abstract}

Keywords: Smart Garbage, Smart AI Dustbin, Segmentation of waste

\section{INTRODUCTION}

Now a days, Waste management is one of the primary problem that the world faces irrespective of the case of developed or developing country. The key issue in the waste management is that the garbage bin at public places gets overflowed well in advance before the commencement of the next cleaning process. It leads to various hazards such as bad odour $\&$ ugliness to that place which may cause for spread of various diseases. To avoid all such hazardous scenario and maintain public cleanliness and health our project is mounted on a smart garbage system. The main aim project is to develop a smart intelligent garbage alert system for a proper garbage management.

\section{DISCUSSION}

The Main Problem that was seen so far was Segregation of Waste, this garbage will segregate the degradable and non-degradable waste using machine learning. The garbage bin is monitored automatically and it will send the information if the garbage bin is full[1]
Tensor Flow Lite is a set of tools to help developers run Tensor Flow models on mobile, embedded, and IoT devices. It enables on-device machine learning inference with low latency and a small binary size. For developers, performing machine learning ondevice can help improve latency, privacy, connectivity, Power consumption.[2]

Mobile Nets are efficient Convolutional Neural Networks for Mobile Vision Applications. Mobile Nets are based on a streamlined architecture that uses depth wise separable convolutions to build light weight deep neural networks[3]

MobileNets is a class of neural, convolutional network designed by Google researchers. They are "mobile-first" coined in that they are built from the ground up to be resource-friendly and run fast, right on our phone. Instead of a single $3 \times 3$ convolution layer accompanied by batch standard and ReLU, the main difference between the MobileNet model and a "traditional" CNN's is MobileNets break the convolution into a $3 \times 3$ depth conv and a $1 \times 1$ pointwise conv.[4]

Mobilenet in its construction, as lightweight. It uses depth-separable convolutions which basically means that on each color channel it executes a single convolution rather than adding all three and flattening it. This has a filtering function of the input channels. For MobileNets a single filter is applied to each input channel by the Depthwise Convolution. The pointwise convolution then applies a $1 * 1$ convolution to combine the depth convolution of the outputs. A standard convolution filters both and in one step combines inputs into a new set of outputs. This is divided into two layers by the depth-separable convolution, a different filter layer and a second layer for blending. This factorisation results in a drastic reduction in calculation and sample scale.[5]

"The Mobilenet's total design is as follows, with 30 layers of 
1. convolutional layer with stride 2

2. depthwise layer

3. pointwise layer that doubles the number of channels

4. depthwise layer with stride 2

5. pointwise layer that doubles the number of channels, etc.[6]

We proposed Logistic Regression for predict the house price targets; the logistic function defined by eq (1)

$$
L R_{k}=\frac{1}{1+e^{-Y_{k} \beta+\varepsilon_{k}}}
$$

Where $L R_{k}$ denote the continuous variable and $Y_{k}$ denote the independent data.[7]

Above function produces the binary outputs that means zero and one, so it is transformed into simple linear regression model by eq (2)

$$
\log \left(\frac{L R_{k}}{1-L R_{k}}\right)=-Y_{k} \beta+\varepsilon_{k}
$$

Finally actual value $L R_{k}$ replaced by other variable $\boldsymbol{L} \boldsymbol{R}_{\boldsymbol{k}}^{*}$; thus we can get the final logistic regression by eq (3)

$$
\log \left(\frac{L R_{k}^{*}}{1-L R_{k}^{*}}\right)=-Y_{k} \beta+u_{k}
$$

where $u_{k}$ is the error factor.

1. The DFD is also called as bubble chart. It is a simple graphical formalism that can be used to represent a system in terms of input data to the system, various processing carried out on this data, and the output data is generated by this system.

2. The data flow diagram (DFD) is one of the most important modeling tools. It is used to model the system components. These components are the system process, the data used by the process, an external entity that interacts with the system and the information flows in the system.

3. DFD shows how the information moves through the system and how it is modified by a series of transformations. It is a graphical technique that depicts information flow and the transformations that are applied as data moves from input to output.

4. DFD is also known as bubble chart. A DFD may be used to represent a system at any level of abstraction. DFD may be partitioned into levels that represent increasing information flow and functional detail.

UML stands for Unified Modeling Language. UML is a standardized general-purpose modeling language in the field of object-oriented software engineering.
The standard is managed, and was created by, the Object Management Group. [8]

Modules here are Image Acquisition, Feature extraction, Classification. Image acquiring is important stage in image processing. Testing image is capturing from Web cam for further process.[9]

In Feature Extraction After the image acquisition, we propose the feature extraction to captured image.in that, we implement deep learning network model like mobile net for feature extraction. It extracts the high level features to image for efficient classification. [10]

The final stage is classification. In that our test Image classified into two categories i.e. Degradable and non-degradable. For Classification, we propose the logistic regression (LR) machine learning method.[11]

\section{CONCLUSION}

A major challenge in waste management is waste disposal which requires segragation of waste into degradable and non degradable categories. Recent advances in computer vision made possible by deep learning has paved the way for AI assisted waste management. This model has certain lacks implementing for bigger bins may be complex, initial implementation may be complex. But this model serves good for segmentation of waste.

\section{REFERENCE}

[1] K. A. Monika, N. Rao, S. B. Prapulla, and G. Shobha et al.( 2016). "Smart dustbin-an efficient garbage monitoring system," vol. 6, no. 6, ( pp. 71137116).

[2] K. M. Kumar et al (2015). "Smart dustbin 1," no. 5, (pp. 101-104).

[3] S. Dugdhe, P. Shelar, S. Jire, and A. Apte et al(2016). "Efficient waste collection system," in 2016 Int. Conf. Internet Things Appl. IOTA 2016, ( pp. 143-147).

[4] J. Joshi et al.(2016), "Cloud computing based smart garbage monitoring system,"( pp. 70-75) .

[5] B. Singh and M. Kaur et al.(2016). "Smart dustbins for smart cities," vol. 7, no. 2, (pp. 610-611)

[6] T. Anagnostopoulos, A. Zaslavsy, A. Medvedev, and S. Khoruzhnicov et al. (2015)."Top - k query based dynamic scheduling for IoT-enabled smart city 
waste collection," in Proc. - IEEE Int. Conf. Mob. Data Manag., vol. 2,( pp. 50-55).

[7] A. D. Deshmukh et al.(2016). "A low cost environment monitoring system using raspberry pi and arduino with zigbee".

[8] S. Mischie et al.(2016). "On teaching Raspberry Pi for Undergraduate University Programmes".

[9] M. Bihis and S. Roychowdhury et al.( 2015). “A generalized flow for multi-class and binary classification tasks: An Azure ML approach," in Proc. - 2015 IEEE Int. Conf. Big Data, IEEE Big Data 2015. (pp. 1728-1737).

[10]Wesley Pereira1, Saurabh Parulekar2, Sopan Phaltankar3 , Vijaya Kamble4 "Smart Bin (Waste Segregation and Optimisation)" Sardar Patel Institute of Technology, BhartiyaVidyaBhavan's campus Munshi Nagar, Andheri-West Mumbai.

[11]A. Chandramohan, J. Mendonca, N. R. Shankar, N. U. Baheti, N. K. Krishnan and M. S. Suma et al.(2014). "Automated Waste Segregator", 2014 Texas Instruments India Edu- cators' Conference (TIIEC), Bangalore, doi: 10.1109/TIIEC.2014.009, (pp. 1-6).

[12] Vishal V, SayantanGangopadhyay - Sir M Visvesvaraya Institute of Technology - "Capacitive Sensors: The Fu- ture of Waste Segregation"

[13] Atmel "8-bit AVR Microcontroller with 32K Bytes In- System Programmable Flash" ATmega328P [Rev No. 7810D-AVR01/15]

[14] Dr Naveen B, Kavya G K, Kruthika S N, Ranjitha K N, Sahana C N - "AUTOMATED WASTE SEGRE- GATOR USING ARDUINO" International Journal of Advance Engineering and Research

[15] Edward A Bruno - "Automated Sorting of Plastics for Recycling"

\section{Reference Article}

India has a 26,000-tonne plastic waste problem http://timesofindia.indiatimes.com/articleshow/67646 745.cms?utm_source=contentofinterest\&utm_mediu m=text\&utm_campaign=cppst

90\% municipal waste in Tamil Nadu dumped without processing https://timesofindia.indiatimes.com/city/chennai/90municipal-waste-in-tamil-nadu-dumped-withoutprocessing/articleshow/65191078.cms 